\title{
DETERMINAÇÃO DOS ELEMENTOS TERRAS RARAS EM AMOSTRAS GEOLÓGICAS POR ICPIAES UTILIZANDO MINICOLUNAS
}

\author{
ROBSON COTA DE OLIVEIRA* \& GERALDO RESENDE BOAVENTURA**
}

\begin{abstract}
RARE EARTH ELEMENTS DETERMINATION IN GEOLOGICAL SAMPLES B Y ICP/AES USING MINICOLUMNS The determination of the rare earth elements (REE) by a simple, quick and low cost method is presented. The method involves sample decomposition, ion-exchange separation using minicolumns and determination of REE ( $\mathrm{La}, \mathrm{Ce}, \mathrm{Nd}, \mathrm{Sm}, \mathrm{Eu}, \mathrm{Gd}, \mathrm{Dy}, \mathrm{Ho}, \mathrm{Er}, \mathrm{Yb}$ and $\mathrm{Lu}$ ) by Inductively Coupled Plasma Atomic Emission Spectrometry (ICP/AES). Geochemical references samples were used for accuracy and precision evaluation. The results are close to the recommended values with relative standard deviations consistem with the literature, demonstrating efficiency, and compatibility with the conventional methods. The recuperation of the heavy REE, Ho, $\mathrm{Er}, \mathrm{Yb}$ and $\mathrm{Lu}$ was $100 \%$; $\mathrm{Nd}, \mathrm{Sm}, \mathrm{Eu}, \mathrm{Gd}$ and $\mathrm{Dy}$ is $98-99 \%$; Ce is $94 \%$ and $\mathrm{La} 85 \%$. The method allows to analyse 18 samples/hour, representing quick and high performance procedures for geological materiais.
\end{abstract}

Keywords: rare earth determinations, ion-exchange, minicolumns, ICP/AES.

RESUMO A determinação dos Elementos Terras Raras (ETR) por um método simples, rápido e de baixo custo é apresentado. O método envolve a decomposição da amostra, separação por troca iônica utilizando minicolunas e leitura dos ETR (La, Ce, Nd, Sm, Eu, Gd, Dy, Ho, Er, Yb e Lu) por Espectrometria de Emissão Atômica com fonte de Plasma (ICP/AES). Amostras de referência geoquímica foram utilizadas para avaliar a exatidão e precisão do método. Os resultados obtidos situam-se próximo aos valores recomendados, com desvios padrão relativo consistentes com os da literatura, demonstrando eficiência e compatibilidade com os métodos convencionais. O percentual de recuperação dos ETR pesados Ho, Er, Yb e Lu foi de 100\%; do Nd, Sm, Eu, Gd e Dy foi de $98 \%$ a $99 \%$; do Ce $94 \%$ e La $85 \%$. O método proposto permite análise de até 18 amostras/hora, representando um procedimento de menor tempo e maior eficiência para materiais geológicos.

Palavras-chaves: determinação de terras raras, troca-iônica, minicoluna, ICP/AES.

INTRODUÇÃO O conhecimento dos teores dos ETR em rochas ou solos é de grande importância em estudos petrogenéticos, metalogenéticos, na prospecção mineral e no entendimento da evolução crustal.

As técnicas instrumentais mais comuns utilizadas na determinação dos ETR são a Ativação Neutrônica, Espectrometria de Massa com Diluição Isotópica, Espectrometria de Emissão Atômica com Plasma Indutivamente Acoplado (ICP/AES ) e Espectrometria de Massa com fonte de Plasma (ICP-MS ). As duas primeiras apesar de apresentarem sensibilidade, exatidão e precisão necessárias na determinação dos ETR em amostras geológicas são de custo e tempo de análise elevados, dificultando a sua aplicação em análises de rotina.

Com o desenvolvimento da Espectrometria de Emissão Atômica com Plasma Indutivamente Acoplado (ICP-AES), reduziram-se os limites de detecção, o efeito matriz e aumentaram a precisão, exatidão, faixa linear dinâmica e o volume de literatura a respeito, demonstrando a eficiência da técnica. $\mathrm{O}$ método normalmente utilizado na determinação destes elementos por ICP/AES é baseado na decomposição da amostra, separação por troca iônica dos elementos da matriz e leitura no instrumento. Os primeiros estudos envolvendo a separação dos ETR em coluna de troca iônica foram realizados por Strelow (1978), e o processo de separação e determinação por ICP/AES tem sido utilizado por diversos autores (Walsh et al 1981, Crock \& Licthe 1982, Dutra 1984, Roelandts 1986, Watkins \& Nolan 1992, Boaventura \& Furtado 1993, Oliveira 1995).

A separação dos ETR por troca iônica é um processo bastante eficiente e conveniente. Os maiores problemas do método tem sido o emprego de colunas com grande quantidade de resina (10a $20 \mathrm{~g}$ ), o que implica em grandes volumes de eluição (300-800 mL), aumentando o tempo e o custo das análises.

Neste trabalho propomos um novo método de separação e determinação dos ETR (La, Ce, Nd, Sm, Eu, Gd, Dy, Ho, $\mathrm{Er}, \mathrm{Yb}, \mathrm{Lu})$, cujas principais características são a simplicidade, rapidez e o custo relativo reduzido em relação aos métodos convencionais. O método baseia-se na utilização de minicolunas e quantidades mínimas de reagentes, proporcionando baixo custo e rapidez analítica.

MÉTODOS Amostras de referência geoquímica Os padrões internacionais JB-3 (basalto), BHVO-1 (basalto), JA-3 (andesito), JG-2 (granito) e JR-2 (riolito) do GSJ (Geological Survey of Japan) e do USGS (United States Geological Survey) foram empregados como referência para acompanhar o desenvolvimento do método e avaliar a sua exatidão.

Decomposição das amostras A decomposição das amostras de referência geoquímica foi feita com abertura ácida convencional para silicatos, com mistura dos ácidos $\mathrm{HC} 1 / \mathrm{HF} / \mathrm{HClO}_{4}$ (Dutra 1984, Totland et al 1992)(Fig 1). Entretanto, esta abertura nem sempre é efetiva para todos os minerais de rochas, principalmente zircão, granada, barita e cromita, resistentes a decomposição ácida. Nestes casos, o resíduo foi filtrado, calcinado, fundido com $\mathrm{LiBO}_{2}$ e disssolvido em $\mathrm{HCl}$ diluído, combinando-se os filtrados. Este procedimento permitiu a decomposição total, das amostras, sem perda dos ETR.

Troca iônica A separação dos ETR foi realizada separadamente com a utilização de uma bomba peristáltica de 6 canais, com velocidade de fluxo ajustável, conectados a minicolunas (Fig 2). A separação dos ETR foi feita com o emprego da resina cationica DOWEX-50Wda Dow Chemical Co, 200-400 mesh e minicolunas plásticas com diâmetro de 6 mm e comprimento de $25 \mathrm{~mm}$ (Fig 3). Uma alíquota da amostra $(5 \mathrm{~mL}$ ou $10 \mathrm{~mL})$ foi eluída com quantidades mínimas de $\mathrm{HNO}_{3}$ 2,0 moles/L (5mL), e HC16,0 moles/L (10 mL) com velocidade de fluxo de $(1,9 \pm 0,1) \mathrm{mL} / \mathrm{min}$.

Instrumentação Todas as medidas foram realizadas em um espectrômetro de emissão atômica com fonte de plasma da marca SPECTROFLAME modelo FMV 05 SCANNER, montagem Paschen-Runge, distância focai de $750 \mathrm{~mm}$, grade côncava com 3600 ranhuras $/ \mathrm{mm}$, faixa espectral de 210 a $480 \mathrm{~nm}$, dispersão recíproca variando de 0,25 a $0,67 \mathrm{~nm} / \mathrm{mm}$.

* Curso de Pós-Graduação em Química Analítica, Departamento de Química da Universidade de Brasília Campus Asa Norte 70910-900 Brasília-DF. / CPMTC / IGC/ UFMG. email: robcogeo@oraculo.lcc.ufmg.br.

** Laboratório de Geoquímica do Instituto de Geociências da Universidade de Brasília, Campus Asa Norte 70910-900 Brasília -DF. email: grbunb@guarany.cpd.unb.br 


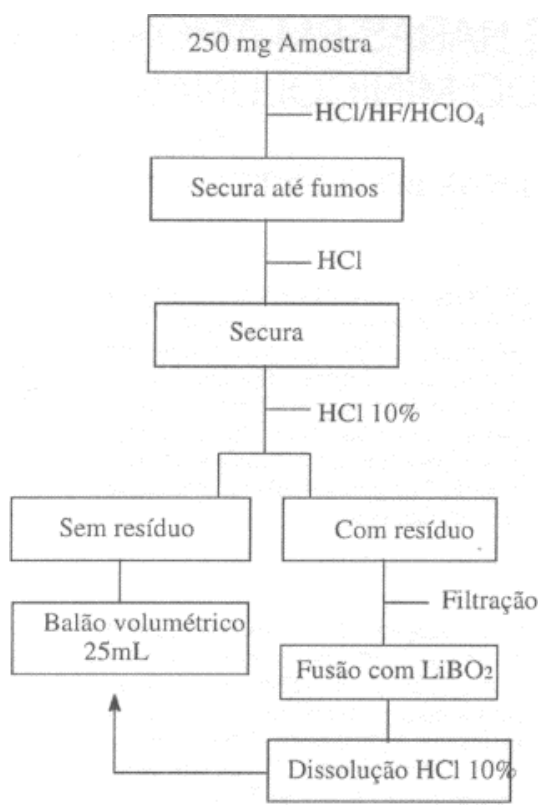

Figura l-Diagrama de procedimentos de decomposição da amostra.

Figure I - Diagrani showing the sample decompositin proceedure.

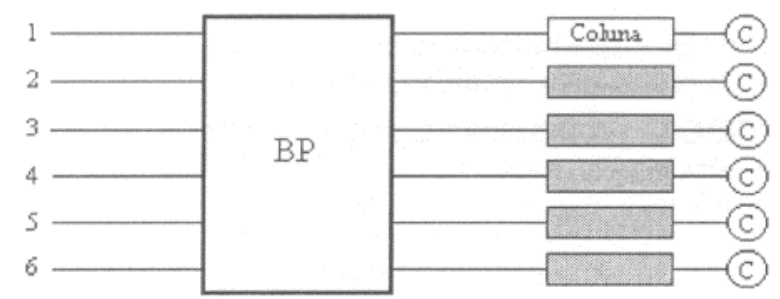

Figura 2 - Diagrama de conexões. B.P =• bomba peristáltica, $C$ - coletor.

Figure 2 - Conexions diagram. B.P. - peristaltic pwnp, C-colector.

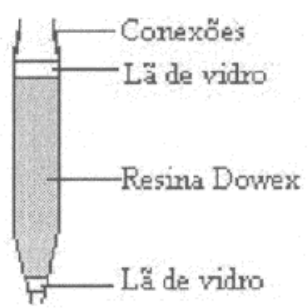

Figura 3 - Minicoluna utilizada no processo de separação por troca iônica.

Figure 3 - Minicolumn used in the ionic exchange separation.

O equipamento possui um policromador específico para os ETR, o que permitiu a determinação simultânea de 11 ETR com pequeno volume de amostra. Os parâmetros operacionais do ICP/AES utilizados neste trabalho se encontram na Tabela 1. Na separação dos ETR foi utilizada uma bomba peristáltica de 6 canais da marca ALITEA C6-XV, com velocidade variável de 2,5-50 rpm.

RESULTADOS E DISCUSSÃO Os limites de detecção e os comprimentos de onda utilizados para cada elemento se encontram na Tabela 2.

A figura 4 mostra o cromatograma completo do processo de separação dos ETR, obtidos a partir de uma solução padrão
Tabela J - Parâmetros operacionais do ICP/AES para análise dos ETR.

Table J - ICP/AES operational setting for REE analysis.

\begin{tabular}{|ll|}
\hline Nebulizador & MEINHARD \\
Fluxo de gás auxiliar & $0,5 \mathrm{~L} / \mathrm{min}$ \\
Fluxo de gás de resfriamento & $14 \mathrm{~L} / \mathrm{min}$ \\
Fluxo de amostra & $2 \mathrm{~mL} / \mathrm{min}$ \\
Pressão do Nebulizador & $2,4 \mathrm{bar}$ \\
Potência do Gerador de RF & $1,2 \mathrm{~kW}$ \\
\hline
\end{tabular}

Tabela 2 - Linhas analíticas e limite de detecção considerando $2 \mathrm{a}$ e intervalo de confiança de $96 \%$.

Table 2 - Analitycal lines and detection limits considering 2a and confidence interval of $96 \%$

\begin{tabular}{|ccc|}
\hline Elemento & $\lambda(\mathrm{nm})$ & $\begin{array}{c}\text { Limite de detecção } \\
(\mu \mathrm{g} / \mathrm{mL})\end{array}$ \\
\hline $\mathrm{La}$ & 398,852 & 0,004 \\
$\mathrm{Ce}$ & 418,660 & 0,02 \\
$\mathrm{Nd}$ & 406,105 & 0,02 \\
$\mathrm{Sm}$ & 259,260 & 0,007 \\
$\mathrm{Eu}$ & 381,967 & 0,001 \\
$\mathrm{Gd}$ & 335,047 & 0,007 \\
$\mathrm{Dy}$ & 353,170 & 0,002 \\
$\mathrm{Ho}$ & 345,600 & 0,003 \\
$\mathrm{Er}$ & 369,265 & 0,003 \\
$\mathrm{Yb}$ & 328,937 & 0,0002 \\
$\mathrm{Lu}$ & 261,542 & 0,0004 \\
\hline
\end{tabular}
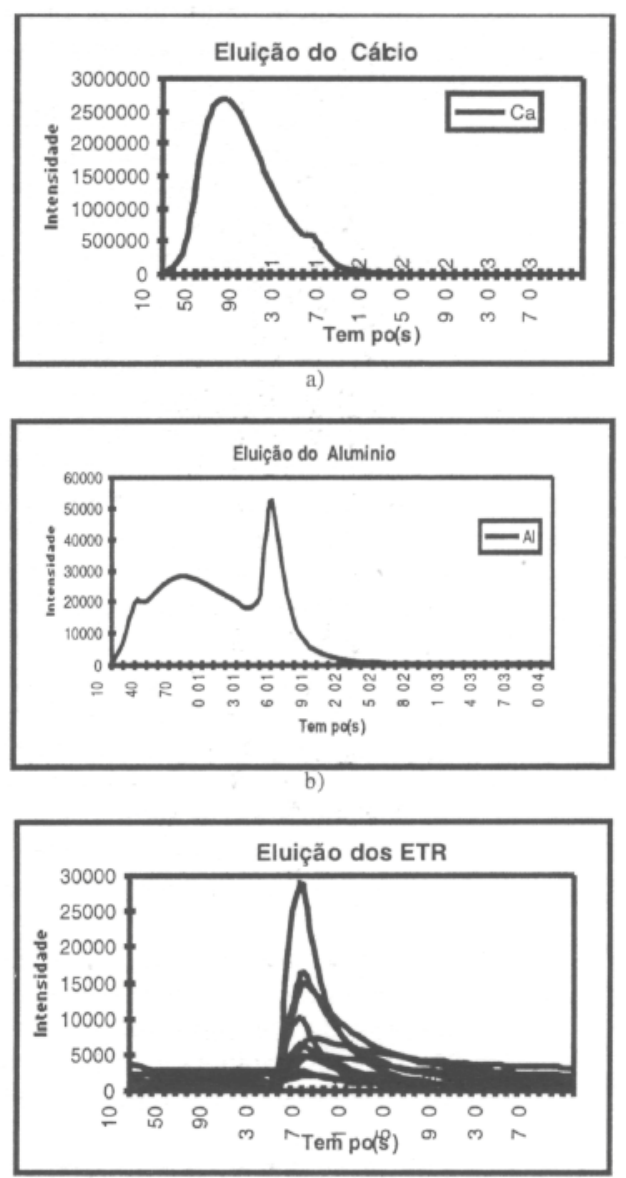

Figura 4 - Cromatograma de eluição obtido do processo de separação: a) eluição do cálcio em $\mathrm{HNO}_{3}$ 2,0 moles/L; b) eluição do alumínio em $\mathrm{HNO}_{3}, 2,0$ moles/L; c) eluição dos ETR em HCl 6,0 moles/L); Fluxo: $(1,9 \pm 0,1) \mathrm{mL} / \mathrm{min}$. Figure 4 - Eluïon chromatogram obtained during the separation: a) elution ofcalcium in $\mathrm{HNO}_{3} 2,0 \mathrm{mols} / \mathrm{L}$; b) elution ofaluminum in $\mathrm{HNO}_{3} 2,0 \mathrm{mols} / \mathrm{L}$; c) elution of REE in $\mathrm{HCl} 6,0 \mathrm{mols} / \mathrm{L})$; Flux: $(3,9 \pm 0, \mathrm{~J}) \mathrm{mL} / \mathrm{min}$. 
multielementar de ETR. A figura 4c registra que, aproximadamente a $130 \mathrm{~s}$, inicia a eluição dos ETR, o que corresponde ao início da passagem de HC16,0 moles/L pela coluna. De 0 a $130 \mathrm{~s}$, os elementos da matriz (rocha), tais como cálcio e alumínio, são separados (Figs 4a e 4b). Neste intervalo de

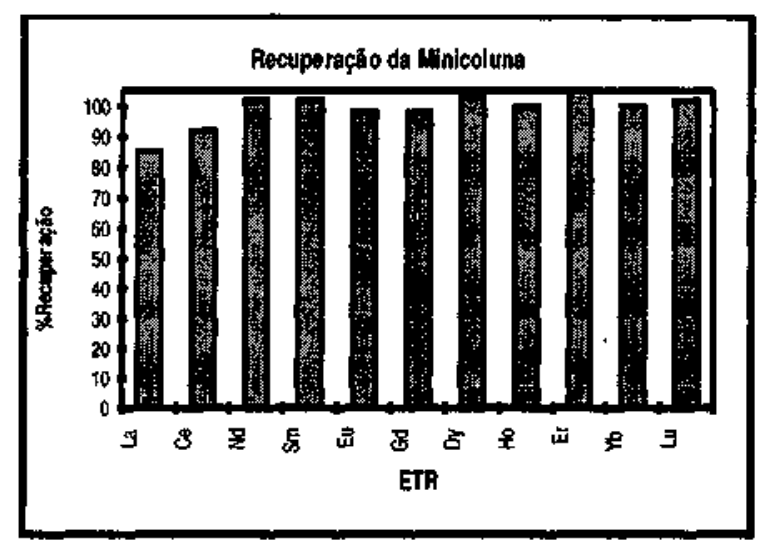

Figura 5 - Porcentagem de recuperação dos ETR na minicoluna.

Figure 5 - Recovery percentage of the REE minicolumn.

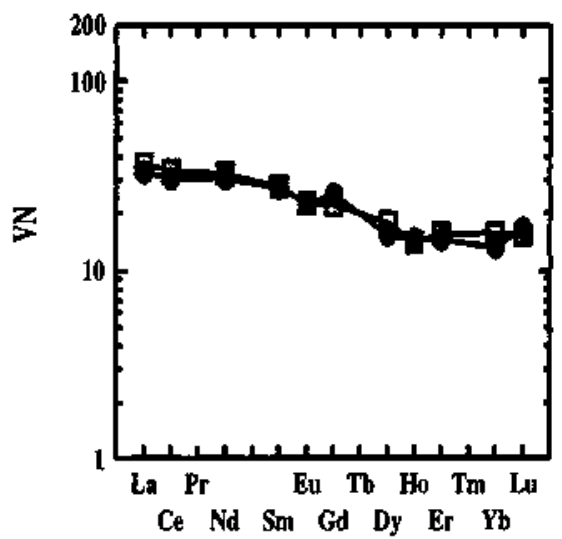

JB-3

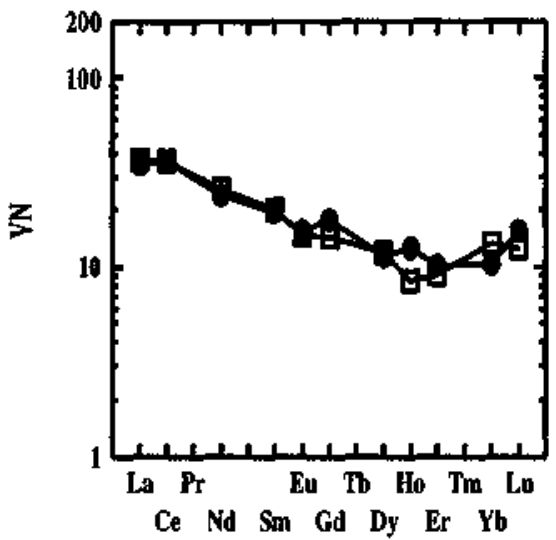

JA-3 tempo ocorre a passagem de $\mathrm{HNO}_{3} 2,0$ moles/L pela coluna, o qual tem o papel de eluir os elementos maiores da rocha.

Para avaliar a eficiência do processo quanto à porcentagem de recuperação da minicoluna (Fig 5) foi empregado um padrão multielementar. A figura mostra que os ETR mais pesados (Ho, Er, $\mathrm{Yb}$ e Lu) são $100 \%$ recuperados. O Nd, Sm, $\mathrm{Eu}, \mathrm{Gd}$ e Dy são recuperados em torno de $98-99 \%$. Os maiores problemas se referem aos elementos Ce (94\%) e o La(85\%). Contudo, a recuperação menor do $\mathrm{La}$, inferior ao valor recomendado, não inviabiliza o método proposto.

Os resultados obtidos (Tabela 3 ) com as 5 amostras de referência geoquímica são bastante próximos aos valores recomendados na literatura, demonstrando a sua compatibilidade com os métodos convencionais. O maior desvio ocorreu com o La, por ser o último ETR a ser eluído. Este problema pode ser contornado de formas alternativas. A primeira seria a introdução de um valor empírico de correção obtido da avaliação da porcentagem de recuperação do La na minicoluna em função da concentração do elemento na amostra compatível com amostras geológicas. Outra seria o aumentar o volume de eluição para $20 \mathrm{~mL}$, com posterior concentração para 5 ou $10 \mathrm{~mL}$. Por último, fazer a eluição dos ETR com $\mathrm{HNO}_{3}$ 6,0 moles/L, aumentando a eficiência de recuperação na minicoluna. $\mathrm{O}$ uso de $\mathrm{HNO}_{3}$ tem as desvantagens de degradar a resina mais rapidamente, custo mais elevado e manuseio mais difícil.

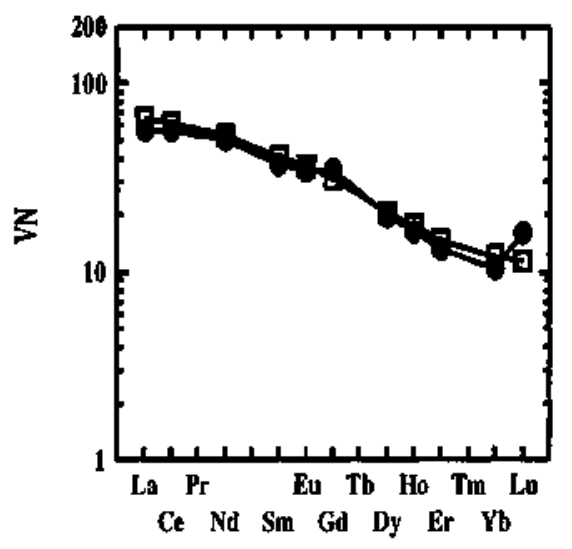

BHV0-1

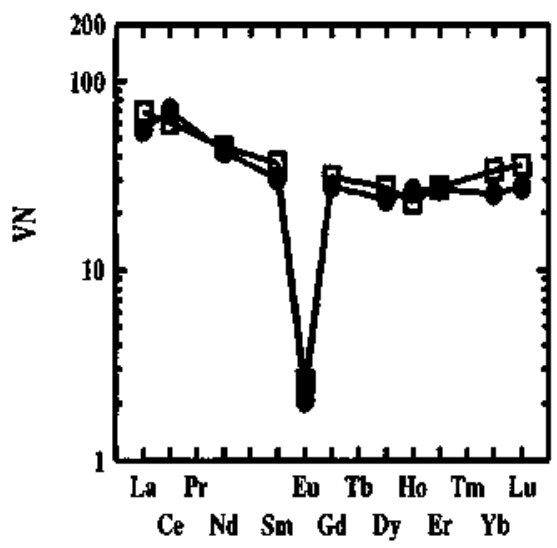

JR-2

Figura 6 - Diagrama dos ETR normalizado ao condrito (Evensen et al. 1978). Os círculos correspondem aos valores obtidos neste trabalho e os quadrados a valores de literatura. $V N=$ valor normalizado.

Figure 6 - Chondrite-normalized REE diagrams (Evensen et al $J$ 978). Circles correspond to values obtainedfor this paper and squares are values from the liietrature. $V N$ - normalized value. 
Tabela 3 - Resultados obtidos para as amostras de referência geoquímica em $\mu \mathrm{g} / \mathrm{g}$.

Table 3 - Results obtained with geochemical reference xamples in $\mu \mathrm{g} / \mathrm{g}$.

\begin{tabular}{|c|c|c|c|c|c|c|c|c|c|c|}
\hline & $\mathbf{L} \mathbf{a}$ & $\mathrm{Ce}$ & Nd & Sm & Eu & Gd & Dy & $\mathbf{E r}$ & $\mathbf{Y b}$ & La \\
\hline $\mathrm{JB}-\mathbf{3}$ & & & & & & & & & & \\
\hline Vexp & $8,1 \pm 0,3$ & $19,5 \pm 0,7$ & $14,5 \pm 0,5$ & $4,2+0,4$ & $1,3 \pm 0,1$ & $5,2 \pm 0,4$ & $3,9+0,2$ & $2,4 \pm 0,2$ & $2,2 \pm 0, \mathrm{~J}$ & $0,42 \pm 0,05$ \\
\hline $\begin{array}{l}\text { Referência } \\
\text { BHVo.1 }\end{array}$ & $8,9 \pm 0,8$ & $21,5 \pm 1,6$ & $15,4 \pm 2,0$ & $4,3 \pm 0,2$ & $1,3 \pm 0,1$ & $4,5 \pm 0,3$ & $4,6 \pm 0,4$ & $2,6 \pm 0,2$ & $2,6 \pm 0,4$ & $0,39 \pm 0,05$ \\
\hline vexp & $13,7 \pm 0,8$ & $35,6 \pm 2,3$ & $23,8 \pm 4,2$ & $5,7 \pm 0,2$ & $2,0 \pm 0,2$ & $7,1 \pm 0,3$ & $5,0 \pm 0.7$ & $2,2 \pm 0,4$ & $1,7 \pm 0,2$ & $0,41 \pm 0,06$ \\
\hline Referencia & $15,8 \pm 1,3$ & $39 \pm 4$ & $25.2 \pm 2,0$ & $6.2 \pm 0.3$ & $2,1 \pm 0,1$ & $6,4 \pm 0.5$ & $5,2 \pm 0,3$ & $2,4 \pm 0,2$ & $2,0 \pm 0,2$ & $0,29 \pm 0,03$ \\
\hline & $8,6 \pm 0,8$ & $23,1 \pm 1,8$ & $11,4 \pm 0,4$ & $3,0 \pm 0,2$ & $0,90 \pm 0,10$ & $3,6 \pm 0,5$ & $2,9 \pm 0,1$ & $1.7 \pm 0.2$ & $1,7 \pm 0,1$ & $0,39 \pm 0,06$ \\
\hline $\begin{array}{l}\text { Referencia } \\
\text { JG-2 }\end{array}$ & $9,0 \pm 1,0$ & $23,3 \pm 2,2$ & $\pm 2.3 \pm 1,0$ & $3,1 \pm 0,2$ & $0,85 \pm 0,08$ & $2,9 \pm 0.7$ & $3.0 \pm 0.5$ & $1,5 \pm 0.5$ & $2.2 \pm 0,3$ & $0,32 \pm 0,07$ \\
\hline vexp & $15,9 \pm 1,8$ & $44,6 \pm 4,0$ & $24,2 \pm 0,7$ & $7,0 \pm 0.1$ & $0,09 \pm 0,05$ & $8,0 \pm 0,8$ & $10,0 \pm 0,6$ & $6.7 \pm 0,7$ & $6,7 \pm 0,4$ & $0,92 \pm 0,11$ \\
\hline $\begin{array}{l}\text { Referencia } \\
\text { JR-2 }\end{array}$ & $20.1 \pm 2,8$ & $49.5 \pm 4.1$ & $25,8 \pm 2,7$ & $7,7 \pm 1,2$ & $0,09 \pm 0.04$ & $7.1 \pm 1.9$ & $11.5 \pm 1.0$ & $5,0 \pm 1,4$ & $7.3 \pm 1.2$ & $1,2 \pm 0,1$ \\
\hline Vexp & & & & 60.6 & & & & & & \\
\hline$f e r$ & & \pm 39 & $21,1 \pm 2,7$ & $5,7 \pm 0,6$ & $0,15 \pm 0,06$ & $6,3 \pm 0,7$ & $6,9 \pm 1,0$ & $4,5 \pm 0,6$ & $5,5 \pm 0,5$ & $0,90 \div 0,0$ \\
\hline
\end{tabular}

Referência Govindaraju( 1994)

Tabela 4 - Comparação de custos e tempo entre o método proposto e os convencionais.

Table 4 - Comparison of costs and time between the proposed and the concentional methods.

\begin{tabular}{|c|c|c|}
\hline \multicolumn{3}{|c|}{ ABERTURA } \\
\hline $\begin{array}{l}\text { Tomada de amostra } \\
\text { Custo de reagentes } \\
\text { Tempo }\end{array}$ & $\begin{array}{l}\text { Normal } \\
0,5-1,0 \mathrm{~g} \\
\text { US } \$(0,60-1,20) \\
16 \text { horas }\end{array}$ & $\begin{array}{l}\text { Método proposto } \\
100 \mathrm{mg} \\
\text { US } \$ 0,20 \\
\text { Red́uçă de } 50 \%\end{array}$ \\
\hline \multicolumn{3}{|c|}{ ELUICAO } \\
\hline $\begin{array}{l}\text { Custo de reagentes } \\
\text { Tempo eluiçä̃o } \\
\text { Tempo conçentraçiso }\end{array}$ & $\begin{array}{l}\text { US } \$(0,75-1,80) \\
4-7 \text { horas } \\
6-16 \text { horas }\end{array}$ & $\begin{array}{l}\text { US\$0,03 } \\
10 \text { minutos( } 6 \text { ) } \\
\text { Leitura direta }\end{array}$ \\
\hline \multicolumn{3}{|c|}{ RESWA } \\
\hline Custo & US\$2-4 & US $\$ 0,10$ \\
\hline
\end{tabular}

Os diagramas de normalização foram construídos e comparados com os valores da literatura (Fig 6). Pode-se observar que os valores obtidos neste trabalho estão muito próximos aos da literatura. Mesmo valores mais baixos para La (sem correção) não comprometem as interpretações geoquímicas/geológicas.

Uma comparação do método apresentado com os métodos clássicos com grandes colunas de separação (Tabela 4) quanto à abertura, eluição e resina, demonstra que tempo de análise e o custo de reagentes são significativamente diferentes. A principal diferença reside na eluição, pois nos métodos clássicos o tempo estimado é da ordem de 2-3 dias e o custo em torno de US\$(0,75-1,80)/amostra, enquanto no método proposto o tempo estimado é da ordem de 10 minutos e custo é da ordem de US\$0,03/amostra.

CONCLUSÕES A aplicação do método proposto para a determinação dos ETR possibilita a obtenção de uma quantidade maior de dados a custo e tempo menores. Os métodos clássicos utilizados em diversos laboratórios são de custo elevado e necessitam tempo excessivo na preparação das amostras para leitura no ICP/AES. Este método, aplicado em análise de rotina dos ETR, permite a determinação de até 18 amostras em tempo médio de 60 minutos, eficiência superior aos métodos convencionais.

No método proposto há uma separação com ou sem préconcentração da amostra, implicando em que sua limitação está diretamente relacionada com os limites de detecção, os quais satisfazem os níveis de concentração comuns em materiais geológicos analisados como basalto, granito, riolito e andesito. As possíveis perdas, principalmente do La, podem ser facilmente contornadas, não comprometendo a aplicação do método em amostras de material geológico. Do ponto de vista geoquímico e petrogenético os resultados apresentados são satisfatórios.

Agradecimentos Ao CNPq pelo apoio financeiro para a realização desta pesquisa e ao Prof. Ricardo Erthal Santelli da Universidade Federal Fluminense pelo apoio e sugestões e a dois revisores anônimos pelas sugestões críticas valiosas.

\section{REFERÊNCIAS}

Boaventura, G.R.; Furtado, J.R. 1993. Determinação de elementos Terras Raras nas amostras de referência geoquímica DNC-1 e W-2 por Espectrometria de Plasma(ICP), após pré-concentração em resina de troca iônica DOWEX 50 8x. IV Congresso Brasileiro de Geoquímica, $279-280$

Clock, J.G. ; Lichte, F. E. 1982. Determination of rare-earth elements in geological materiais by inductively coupled argon plasma/atomic emission spectrometry. Anal. Chem., 54: 1329-1333.

Dutra,C.V. 1984. Método para determinação de traços e sub-traços de Terras Raras em rochas por Espectrometria de Plasma(ICP)- aplicação em petrogênese. XXXIII Congresso Brasileiro de Geologia. Anais: 4792-4805.

Evensen, N.M.; Hamilton, P.J.; O'nions, R. K. 1978. Rare earth abundances in chondritic meteorites. Geochim. Cosmochim. Acta, 42: 1199-1212.

Govindaraju, K.(1994). 1994. Compilation of working values and sample description for 383 geostandars. Geostand. Newsl., 18(special issue): $1-158$.

Oliveira, R.C. 1995. Análise Química de Terras Raras em amostras candidatas a referência geoquímica: PM-S e WS-E utilizando-se ICP/EEA. VIII Encontro Nacional de Química Analítica, 221.
Roelandts, I. 1986. Seqüencial inductively coupled plasma determination of some rare-earth elements in five french geostandards. Geostand. Newsl., 10(2): 135-154

Strelow,F.W.E.; Victor, A.H.;Weinart, C.H.S.W. 1978. lon exchange chromatography applied to the separation methods and accurate determination of some trace elements in rocks. Geostand. Newsl., 2(1): 49-55.

Totland, M.; Jarvis, L; Jarvis, K. E. 1992. An assessment of dissolution techniques for analyses of geological samples by plasma spectrometry. Chem. Geol. 95: 35-62.

Walsh, J.N.; Buckley, F.; Barker J. 1981. The simultaneous determination of the rare-earth elements in rocks using inductively coupled plasma source spectrometry. Chem. Geol. :33: 141-153.

Watkins, P.J.; Nolan, J. 1992. Determination of rare-earth element, yttrium, scandium and hafnium using cation-exchange separation and inductively coupled plasma-atomic emission spectrometry. Chem. Geol,, 95:131-139.

Manuscrito A-956 Recebido em 26 de fevereiro de 1998 Revisão dos autores em 20 de junho de 1998 Revisão aceita em 25 de junho de 1998 